\title{
Spinal cord MRI in multiple sclerosis with multicoil arrays: a comparison between fast spin echo and fast FLAIR
}

\author{
Massimo Filippi, Tarek A Yousry, Hatem Alkadhi, Michael Stehling, Mark A Horsfield, \\ Raymond Voltz
}

\begin{abstract}
Objectives-To compare the sensitivity of fast spin echo (FSE) and of fast fluid attenuated inversion recovery (fast FLAIR) in detecting spinal cord lesions in multiple sclerosis.

Methods-With a 1.5 Tesla machine and a multicoil receiver array, FSE images (with two different pixel sizes) and fast FLAIR images of the spinal cord were obtained from 13 patients with multiple sclerosis.

Results-Twenty three lesions (10 cervical, 12 thoracic, and one lumbar) were found in seven patients (54\%) using FSE with the larger pixel size. Seventeen lesions (seven cervical and 10 thoracic) were detected in the same seven patients using FSE with smaller pixel size. Nine lesions (five cervical and four thoracic) were found using fast FLAIR in six patients (46\%). All the lesions found using fast FLAIR were detected using the other two techniques and all the lesions detected by FSE with smaller pixel size were detected using FSE and greater pixel size.

Conclusion-Fast FLAIR sequences detect substantially fewer cord lesions in patients with multiple sclerosis.
\end{abstract}

(F Neurol Neurosurg Psychiatry 1996;61:632-635)

Keywords: multiple sclerosis; magnetic resonance imaging; spinal cord; fast spin echo; fast fluid attenuated inversion recovery

Recent developments in MRI techniques have dramatically improved the resolution of spinal cord MRI. In multiple sclerosis, this achievement is of particular interest, as the ability to image reliably lesions within the spinal cord may have a major impact on the understanding of the pathophysiology of the disease. Using a multicoil receiver array and fast spin echo (FSE) sequences, it is now possible to demonstrate spinal cord lesions in about $75 \%$ of patients with clinically definite multiple sclerosis $^{1}$ and to exclude other possible causes of spinal cord damage in patients who are suspected clinically of having the disease. ${ }^{2}$
Fluid attenuated inversion recovery (FLAIR) sequences produce heavily T2 weighted images with suppression of CSF signal by combining a long inversion time inversion recovery sequence with a long echo time. The fast FLAIR sequences have greatly improved the sensitivity of MRI for the detection of multiple sclerosis lesions in the brain. ${ }^{35}$ Both the number of lesions and their total volume are larger in fast FLAIR images of the brain compared with conventional spin echo. Using surface coils, FLAIR was shown to be more sensitive than conventional spin echo sequences in detecting multiple sclerosis lesions in the cervical cord ${ }^{67}$ The suppression of the signal from the CSF was thought to improve the sensitivity of spinal cord imaging by reducing CSF related artefacts which potentially mask the lesions. The time saving given by fast FLAIR compared with standard FLAIR makes fast FLAIR much more attractive for practical applications. Similarly, it was thought that improvements in the inplane resolution made possible by the FSE sequence would also lead to an increase in sensitivity for detecting intrinsic cord lesions. ${ }^{2}$ The use of multicoil arrays also allows images of the whole spinal cord to be obtained with good signal to noise ratio and resolution.

The aim of this study was to compare the sensitivities of fast FLAIR and FSE with different inplane resolutions in detecting spinal cord lesions in patients with multiple sclerosis.

\section{Patients and methods \\ PATIENTS}

Thirteen (five men and eight women) consecutive outpatients with clinically definite multiple sclerosis $^{8}$ entered the study. Nine had relapsing-remitting and four had secondary progressive multiple sclerosis. The mean age was 34.4 (SD 9.3) years, the median duration of the disease was four (range 1-14) years, and the median expanded disability status scale $\left(\right.$ EDSS) ${ }^{9}$ was $2 \cdot 0$ (range $0-7 \cdot 0$ ). Patients who had experienced an acute relapse or who had taken steroids during the preceding six months were excluded from the study. Written informed consent was obtained from all the patients before inclusion. 
MRI

All MRI was were carried out using a 1.5 Tesla superconducting system (Vision, Siemens, Erlangen, Germany). The receiver coil array consisted of six separate coils, although only four of the elements were active. The slice thickness was $3 \mathrm{~mm}$, with a field of view (FOV) greater than $500 \mathrm{~mm}$ in the superior-inferior direction in all cases, enabling the entire cord to be visualised. For the fast FLAIR images, two separate acquisitions were necessary to give enough slices to completely cover the spinal cord; these two acquisitions were therefore performed with alternating slices interleaved. A total of nine slices was obtained for all the sequences used. The image acquisition parameters were as follows:

(1) FSE with larger pixel size: TR = $3817 \mathrm{~ms}, \mathrm{TE}=112 \mathrm{~ms}$, echo train length $=$ 15. The phase encoded direction was superior-inferior with $\mathrm{FOV}=655 \times 500$ (phase encode $\times$ readout), raw data matrix $=$ $512 \times 512$, pixel size $=1.279 \times 0.976 \mathrm{~mm}$ (phase encode $\times$ readout), number of signal averages $=1$, acquisition time $=2 \min 13 \mathrm{~s}$. This sequence incorporated gradient moment nulling (flow compensation) in the readout and slice selection directions. The short acquisition time for this sequence allowed the phase encoding to be in the superior-inferior direction, thus reducing the possibility of motion related artefacts from being superimposed on the spinal cord. Phase encoding oversampling was used to remove the wrap artefact, and the final image was displayed with $500 \times 500 \mathrm{~mm}$ FOV.

(2) FSE with smaller pixel size: $\mathrm{TR}=$ $5000 \mathrm{~ms}, \mathrm{TE}=112 \mathrm{~ms}$, echo train length $=$ $15, \mathrm{FOV}=500 \times 500$, raw data matrix size $=$ $720 \times 1024$, pixel size $=0.694 \times 0.488 \mathrm{~mm}$ (phase encode $\times$ readout), number of signal averages $=1$, acquisition time $=4 \min 5 \mathrm{~s}$. This sequence incorporated gradient moment nulling only in the readout direction. Phase encoding was anterior-posterior.

(3) fast FLAIR sequence: TR $=9000 \mathrm{~ms}$, $\mathrm{TE}=105 \mathrm{~ms}$, TI $=2200 \mathrm{~ms}$, echo train length $=7, \quad \mathrm{FOV}=250 \times 500 \mathrm{~mm}$, raw data matrix size $=210 \times 512$, pixel size $=$ $1.190 \times 0.976 \mathrm{~mm}$ (phase encode $\times$ readout), number of signal averages $=1$, total acquisition time $=9 \mathrm{~min} 18 \mathrm{~s}$. This sequence had no gradient moment nulling. Phase encoding was anterior-posterior.

The TR, TE, and TI we used are similar to those of a previous comparative study with conventional spin echo and FLAIR. ${ }^{6}$ However, there were some implementational differences; in particular, the fast FLAIR sequence we used incorporated slice selective inversion pulses, whereas the FLAIR sequence used by Thomas et $a l^{6}$ used non-selective inversion

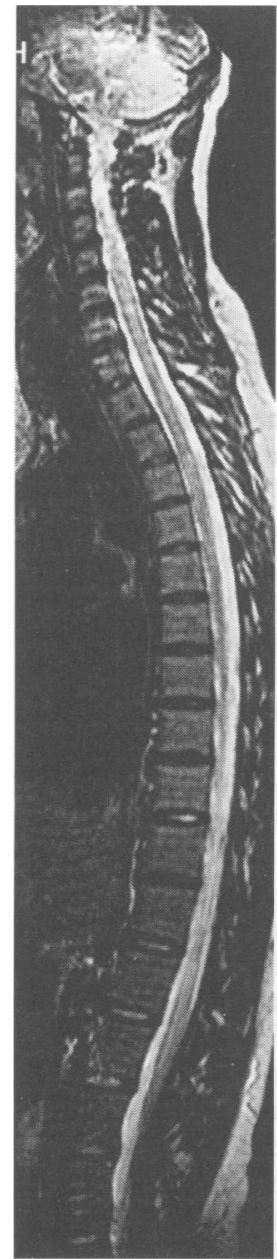

A

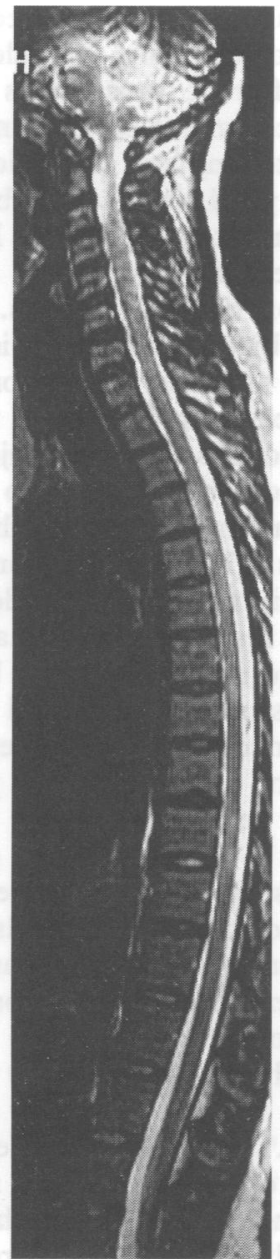

B

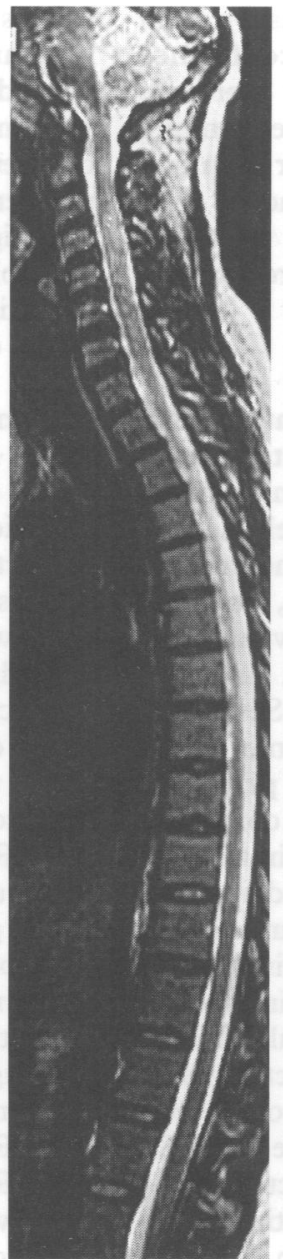

C

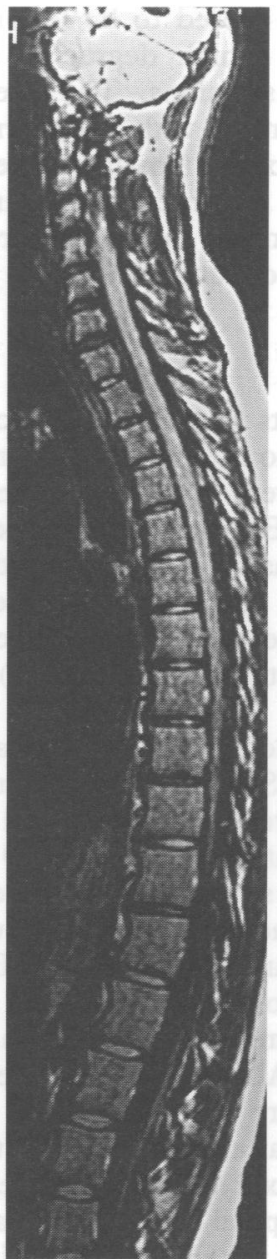

$D$

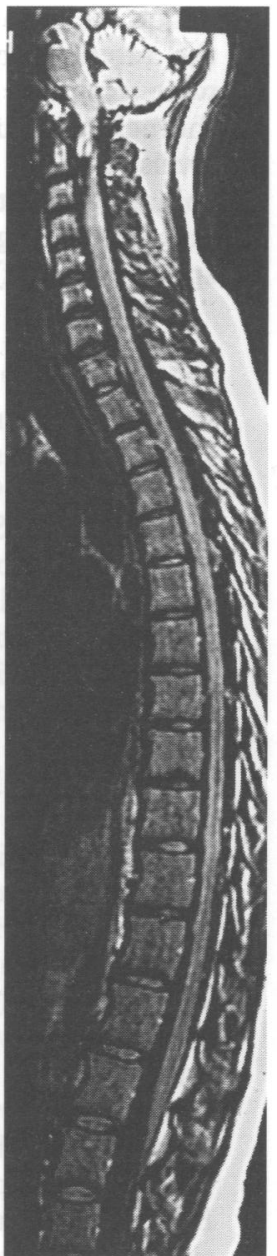

E

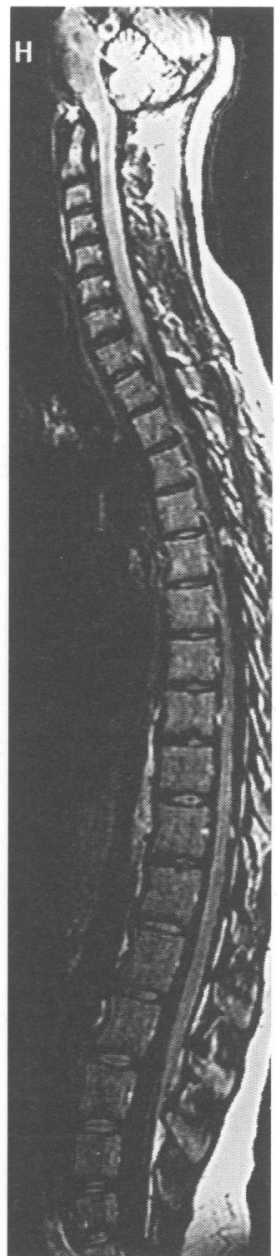

F

Contiguous coronal slices obtained with FSE and large pixel size (A-C) and with fast FLAIR (D-F) in patient 2 (see table). In A-C many intrinsic lesions are seen both in the cervical and thoracic cord; only few of them are seen in D-F (see table for further details). 


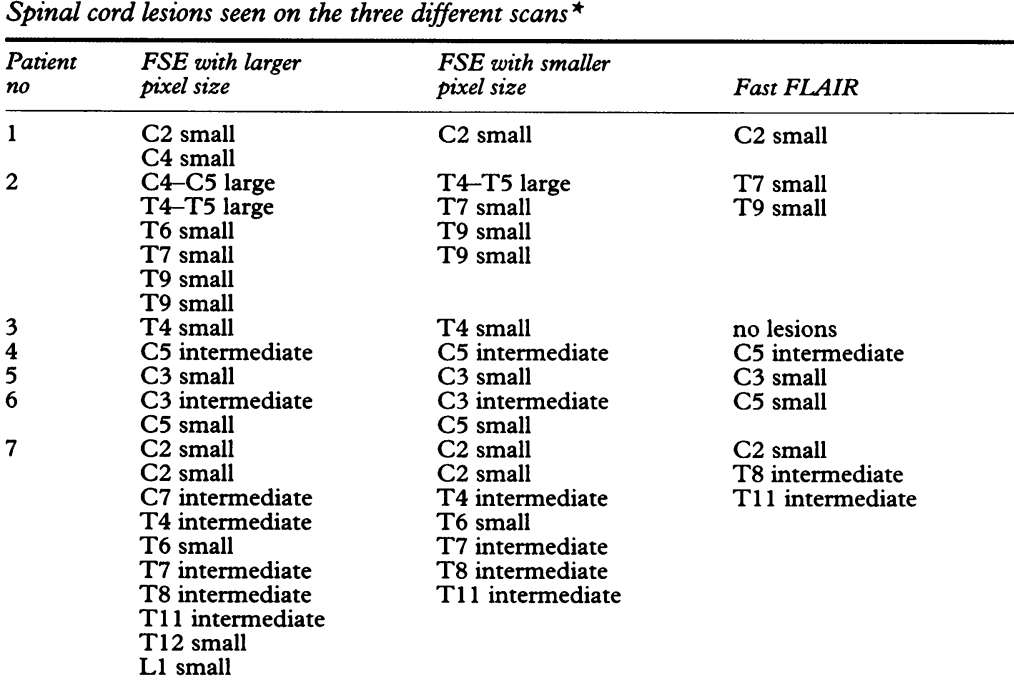

*Only patients with at least one lesion in one of the three sequences are reported. See text for further details. pulses with the result that CSF suppression was optimal only in the central slice of their 12 slice data set.

Two of us (MF and TAY) evaluated the number, size, and location of lesions by consensus. Firstly, the fast FLAIR and the two sets of FSE sequences for all the patients were evaluated in a random order. Next, for each patient, the different sequences were compared side by side. Only hyperintense areas which were considered to be lesions by both the raters with a high degree of confidence were counted as lesions. The site (cervical, thoracic, or lumbar with the corresponding levels) and the extent (small: smaller than one spinal segment; intermediate: extending over one spinal segment; large: longer than one spinal segment) of each lesion were recorded.

\section{Results}

Table 1 presents the number, location, and size of cord lesions for each patient. Twenty three lesions were found in seven patients (54\%) using FSE with the larger pixel size (figure). Ten lesions were located in the cervical cord (six small, three intermediate, and one large), 12 in the thoracic cord (seven small, four intermediate, and one large), and one small lesion in the lumbar cord. Seventeen lesions were detected in the same seven patients using FSE with the smaller pixel size. Seven lesions were in the cervical cord (five small and two intermediate) and 10 in the thoracic cord (five small, four intermediate, and one large). Nine lesions were found in six patients (46\%) using fast FLAIR. Five lesions were cervical (four small and one intermediate) and four were thoracic (two small and two intermediate). All the lesions detected using fast FLAIR were seen using the other two techniques and all the lesions detected by FSE with smaller pixel size were detected using FSE and larger pixel size. In one patient, considered to have no abnormalities in the cord when fast FLAIR was used, one small lesion located at T4 was detected by both the FSE sequences.

\section{Discussion}

A complete assessment of spinal cord lesions in multiple sclerosis may have a major impact on the understanding of the pathophysiology of the disease. It is indeed conceivable that the lesions located in the spinal cord may be most relevant in determining severe physical disability, thus explaining the clinical/MRI discrepancy found in previous studies comparing brain MRI lesion load on T2 weighted scans and disability measured with the EDSS score. ${ }^{10}$

Recently, new sequences (magnetisation transfer gradient recalled echo, FSE with better inplane resolution, and FLAIR) have been proposed to increase further the sensitivity of MRI in detecting intrinsic spinal cord lesions. ${ }^{26711}$ Contrary to expectations, our study, which is the first carrying out a systematic comparison between FSE and fast FLAIR using a multicoil array in patients with multiple sclerosis, shows that fast FLAIR and FSE with smaller pixel size detect fewer spinal lesions compared with FSE with larger pixel size. Whereas there were some implementation differences between these three sequences, it is not thought that these could account for our findings. For example, the lower resolution FSE sequence incorporated gradient moment nulling in the readout and slice selection directions, and thus we might expect this to lead to more false positive lesions in the other two sequences because of flow artefacts; this was clearly not the case. The different sensitivities of the two FSE sequences might be explained by the fact that the better inplane resolution obtained with a smaller pixel size is offset by the poorer resultant signal to noise ratio. In addition, use of multiple echo acquisition may influence the detection of small objects. ${ }^{12}$ The intensity of small objects and edges is enhanced in the phase encoded direction on multiecho sequences with long echo times. Because the spinal cord is a narrow object, the orientation of the cord with respect to the phase encoded direction may influence the severity of edge artifacts. Thus when the long dimension of the cord is in the phase encoded direction (as for the low resolution FSE images) the severity of the edge artefacts should be reduced. These artefacts may mask cord lesions when the phase encoded direction lies across the cord.

On the other hand, it is perhaps more difficult to explain why the sensitivity of fast FLAIR is inferior to that of FSE. There are three aspects of the image acquisition which might go some way towards accounting for the poor performance of the fast FLAIR sequence used here. Firstly, it is possible that a longer echo time might improve FLAIR sensitivity, although the echo time we used was similar to that in a previous study with conventional FLAIR. ${ }^{6}$ The optimum echo time for fast FLAIR of the cerebrum in multiple sclerosis is somewhat longer than that used by us. ${ }^{313}$ Secondly, as spinal cord relaxation times are different from those in the brain, ${ }^{14}$ it may be that a shorter TI would result in better lesion detection, even at the expense of some com- 
promise on CSF suppression. ${ }^{15}$ Thirdly, because of the CSF suppressing properties of the fast FLAIR sequence, the edges of the cord are sharper, and the edge enhancement artefact noted above may be more severe, potentially further masking the internal features of the cord. It was not possible to make the phase encoding direction superior-inferior for either the fast FLAIR sequence or the high resolution FSE sequence, as this would have led to an intolerably long acquisition time. However, it might be expected that the lack of gradient moment nulling could result in flow artefacts on the fast FLAIR images. The fact that fewer lesions were seen on fast FLAIR images suggests that there were no "false" lesions resulting from flow artefacts. It may be that, because of the CSF suppression in fast FLAIR, the use of flow compensation is unnecessary.

These sequence related aspects, however, are probably not enough to explain the greatly reduced sensitivity of fast FLAIR. We know, from previous experience with the brain, that the sensitivity of fast FLAIR compared with conventional spin echo is variable in different anatomical regions: fast FLAIR is much more sensitive than conventional spin echo in detecting cortical/subcortical lesions, whereas the reverse is true for lesions located in the posterior fossa. ${ }^{5}$ It is therefore conceivable that in CNS areas located below the tentorium, for reasons which remain unknown, multiple sclerosis lesions may have intrinsic characteristics different from those located in the supratentorial brain. The MRI properties of the lesions may therefore be very different and the pulse sequences would have to be further optimised for these areas. If these speculations are confirmed by further studies, the different behaviour of multiple sclerosis lesions when different sequences are applied might lead to a better understanding of the pathophysiology of the disease. On the other hand, lesions in the posterior fossa and spinal cord may, because of their location, play an important part in the development of disability in multiple sclerosis. The poor sensitivity of fast FLAIR in these areas is of serious concern if it is to be used to monitor the natural evolution of the disease, or the effect of treatment.

This work derives in part from the collaboration made possible by the EC funded (ERBCHRXCT 940684) European Magnetic Resonance Network in Multiple Sclerosis (MAGNIMS).

1 Kidd D, Thorpe JW, Thompson AJ, et al. Spinal cord MRI using multi-array coils and fast spin echo. II. Findings in using multi-array coils and fast spin echo. II.

2 Thorpe JW, Kidd D, Kendall BE, et al. Spinal cord MRI using multi-array coils and fast spin echo. I. Technical aspects and findings in healthy adults. Neurology 1993 43:2625-31

3 Rydberg JN, Hammond CA, Grimm RC, et al. Initial experience in MR imaging of the brain with a fast fluid-attenuated inversion-recovery pulse sequence. Radiology 1994 193:173-80.

4 Hashemi RH, Bradley WG, Chen DY, et al. Suspected multiple sclerosis: MR imaging with a thin-section fast FLAIR pulse sequence. Radiology 1995;196:505-10.

5 Filippi M, Yousry T, Baratti C, et al. Quantitative assessment of MRI lesion load in multiple sclerosis: a comparison of conventional spin-echo with fast-fluid-attenuated inversion recovery. Brain 1996;119:1349-55.

6 Thomas DJ, Pennock JM, Hajnal JV, et al. Magnetic resonance imaging of spinal cord in multiple sclerosis by fluid-attenuated inversion recovery. Lancet 1993;341:

7 White SJ, Hajnal JV, Young IR, Bydder GM. Use of fluidattenuated inversion-recovery pulse sequences for imaging the spinal cord. Magn Reson Med 1992;28:153-62.

8 Poser CM, Paty D, Scheinberg L, et al. New diagnostic criteria for multiple sclerosis: guidelines for research protocols. Ann Neurol 1983;13:227-31.

9 Kurtzke JF. Rating neurologic impairment in multiple sclerosis: an expanded disability status scale (EDSS). Neurology 1983;33:1444-52.

10 Filippi M, Horsfield MA, Tofts PS, et al. Quantitative assessment of MRI lesion load in monitoring the evolution of multiple sclerosis. Brain 1995;118:1601-12.

11 Finelli DA, Hurst GC, Karaman BA, et al. Use of magnetization transfer for improved contrast on gradient-echo MR images of the cervical spine. Radiology 1994;193: $165-71$

12 Constable RT, Gore JC. The loss of small objects in variable TE imaging: implications for FSE, RARE, and EPI. Magn Reson Imaging 1992;28:9-24.

13 Rydberg JN, Riederer SJ, Hammond CA, Jack CR Contrast optimization in fluid attenuated inversion recovery (FLAIR) imaging. Magn Reson Med 1995;34: 868-77.

14 Ho PSP, Shivei Y, Czervionke LF, et al. MR appearance of gray and white matter at the cervicomedullary region. GFNR Am $\mathcal{F}$ Neuroradiol 1989;10:1051-5.

15 Baratti C, Barkhof F, Hoogenraad F, Valk J. Partially saturated fluid attenuated inversion recovery (FLAIR) sequences in multiple sclerosis: comparison with fully relaxed FLAIR and conventional spin-echo. Magn Reson Imaging 1995;13:513-21. 